\title{
Imaging of high-energy electron beam profile with optical diffraction radiation
}

\author{
Dao Xiang, ${ }^{*}$ Wen-Hui Huang, and Yu-Zheng Lin \\ Department of Engineering Physics, Tsinghua University, Beijing, China, 100084
}

(Received 8 January 2007; published 13 June 2007)

\begin{abstract}
Optical transition radiation (OTR) has been widely used in electron beam profile imaging. Optical diffraction radiation (ODR) has recently been used to measure the electron beam's transverse size with the angular distribution. Because of the close relationship between OTR and ODR, it is natural to ask whether ODR could be used to image the beam profile as is done with OTR. In this paper, the image formation process is investigated as a two-dimensional (2D) convolution. The image formed with ODR as a single electron passes through a circular aperture, through a rectangular slit, and beneath a semi-infinite plane is studied from first principle and taken to be the point spread function (PSF) of the imaging system. It is found that, unlike the OTR case, the PSF of ODR is space variant and largely depends on the shape of the ODR target. With this characteristic, the beam image formed with ODR differs greatly from the real beam profile, and the deconvolution process is generally needed in order to retrieve the real beam distribution from the ODR image. The possibility of using an image formed with ODR from a rectangular slit and a semi-infinite plane to determine beam profile in the direction parallel to the edge of the slit or plane and monitoring the beam's position are estimated. The theoretical prediction is compared to recently reported experimental results and a qualitative agreement is achieved.
\end{abstract}

DOI: 10.1103/PhysRevSTAB.10.062801

PACS numbers: 29.27.Fh, 41.60.- $\mathrm{m}, 41.75 . \mathrm{Ht}$

\section{INTRODUCTION}

The International Linear Collider (ILC) and x-ray free electron lasers (XFELs) require high-energy, high-current, and low-emittance beams. The beam size is a basic parameter for estimating the status of the accelerator, and the beam size measurement is essential for developing such facilities and monitoring instabilities.

Based on long established theories and high quality detectors that work in the optical band, optical transition radiation (OTR) has been widely used for beam transverse profile measurement with energy ranging from $80 \mathrm{keV}$ [1] to $30 \mathrm{GeV}$ [2]. The main drawback of beam profile measurement with OTR is that it is an intercepting method for which the beam directly hits the target. Such direct interaction, on the one hand, causes huge heat deposition to the target and, on the other hand, results in emittance growth by randomly increasing the beam divergence from Coulombic scattering. For the proposed ILC, the high heat deposition associated with the high-energy and high density beam may exclude the use of the intercepting method to measure the electron beam size [3]. As for the XFELs where the beam intensity is not dramatically high, although using OTR to measure beam profile is still a routine, it cannot be used to real-time monitor the beam size in the long undulator, which may be very helpful in preserving overlapping between the self-amplified x-ray and the driving electron beam.

Because of the close relationship between optical diffraction radiation (ODR) and OTR [4], and the fact that ODR is a nonintercepting method which effectively avoids

\footnotetext{
*Author to whom correspondence should be addressed. xiangdao@tsinghua.org.cn
}

heat deposition and emittance degradation due to Coulombic scattering, it is natural to ask whether ODR can be used to image the beam profile as is done with OTR. While using the angular distribution of ODR to measure electron beam size has been extensively studied [5-10], the feasibility of using ODR to image beam profile remains largely undeveloped. The main drawback of beam size determination with ODR angular distribution is that generally both the beam size and divergence contributes to the angular distribution. Theoretical studies have been done which show how the divergence and beam size effects can be separated $[6,8,10]$. However, up to now the angular distribution of ODR has only been employed to measure beam size for cases when the divergence effect is negligible [9]. Likewise, the method presumes a Gaussian distribution for the beam profile and thus only predicts the rms beam size instead of the detailed profile which is typically obtained by direct imaging.

In this paper, the image formation process is investigated as a $2 \mathrm{D}$ convolution. The image formed with ODR as a single electron passes through a circular aperture, through a rectangular slit, and eventually beneath a semi-infinite plane is studied and taken to be the point spread function (PSF) of the imaging system. The image for a whole beam is obtained by convoluting the real beam distribution with the PSF. It was found that, unlike the OTR case for which the characteristic width of PSF was small and the PSF was independent of the transverse position, in contrast, that of ODR was space variant with a characteristic width similar to the impact factor, i.e., the minimum distance from the electron to the ODR target. Generally, there is a significant difference between the beam images formed with ODR from the real beam profile. Moreover, the deconvolution process is needed in order to retrieve the real beam distri- 
bution from the image. The theoretical prediction is compared to experimental results that have been recently reported [11-14], and qualitative agreement is achieved. The possibility of using an image formed with ODR from a rectangular slit and semi-infinite plane to determine beam profile in the direction parallel to slit or plane edge and monitoring the beam position variation are also estimated.

The method of direct imaging with ODR may be complementary to the use of angular distribution. The former can predict the beam profile in the direction parallel to the slit edge, while the latter can predict the beam size in the direction perpendicular to the slit edge [5-10].

The remainder of this paper is organized as follows: Section II discusses the model for calculating PSF and concentrates on imaging with ODR from a circular aperture. Section III studies the image formed with ODR from a rectangular slit and shows how the image could be used to monitor beam position and determine the accurate beam profile. Section IV briefly discusses imaging with ODR from a semi-infinite plane and suggests some scheme for experimental demonstration. The conclusions and discussions are then summarized in Sec. V.

\section{MODEL FOR CALCULATING PSF}

The image formation process can be understood as a $2 \mathrm{D}$ convolution. We follow a standard method to find the image of a beam, which is to first obtain the PSF for the specific imaging system, and then to convolve the real beam distribution with the PSF. As for imaging with ODR, the PSF should only involve the image of a single electron.

The field of a uniformly moving electron can be replaced with the field of pseudophotons. When transmitted through or reflected by the metallic target, the pseudophotons convert to real photons propagating along the direction of velocity and the specular reflection direction. The backward DR is generally extracted out and further measured in the focal plane where the angular distribution is recorded and used to predict the beam size and divergence [5-10]. Alternatively, the detector could be put in the image plane. What the features of the image are and whether it could be used to predict beam profile is the main concern of this paper.

The image of a single electron formed with OTR has been extensively studied [15-19]. The detailed procedures adopted in this paper to calculate the image of a single electron in the image plane can be found in $[18,19]$. Here we just present the main calculation process and important results for completeness. The geometry for calculating the image is shown in Fig. 1. The variables in the source plane, in the lens plane, and in the image plane are denoted by $s, l$, and $i$ in the subscripts, respectively. The $z$-axis of the coordinates is chosen to be the central line of the circular aperture, and the electron is assumed to center pass through the circular aperture. The distances from the radiator to the

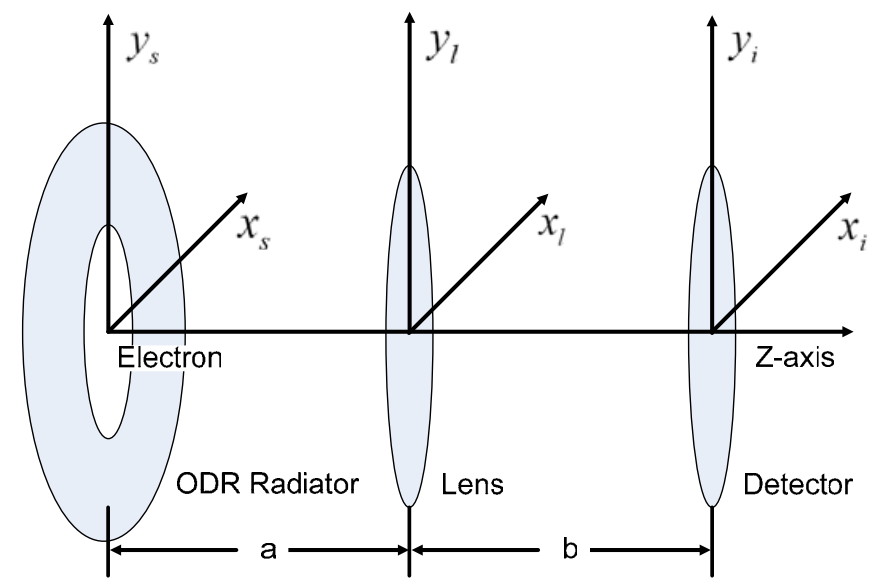

FIG. 1. (Color) Geometry for imaging with ODR from the circular aperture.

lens and from the lens to the detector are chosen to satisfy the imaging condition $1 / a+1 / b=1 / f$, where $f$ is the focal length of the lens.

According to [20], the pseudophoton field for highenergy electron can be written as

$$
E_{x_{s}, y_{s}}^{s}=\frac{e \alpha}{\pi v} \frac{x_{s}, y_{s}}{\sqrt{x_{s}^{2}+y_{s}^{2}}} K_{1}\left(\alpha \sqrt{x_{s}^{2}+y_{s}^{2}}\right),
$$

where $v$ is the velocity of the electron, $\alpha=\omega / v \gamma, \omega$ is the angular frequency, $\gamma$ is the Lorentz factor, and $K_{1}(x)$ is the first-order modified Bessel function of the second kind.

The pseudophotons on the ODR radiator could be considered to comprise a secondary wave source, and the field on the lens can be obtained with the Fresnel diffraction integral. After taking into account the phase shift introduced by the lens and again applying the Fresnel diffraction integral, the field distribution in the image plane for a single electron could be obtained [19]:

$$
\begin{aligned}
E_{x_{i}, y_{i}}^{i}= & -\frac{e \alpha}{\pi \lambda^{2} a b v} \int d x_{l} d y_{l} \int d x_{s} d y_{s} \frac{\left(x_{s}, y_{s}\right)}{{\sqrt{x_{s}{ }^{2}+y_{s}^{2}}}^{2}} \\
& \times K_{1}\left(\alpha \sqrt{x_{s}{ }^{2}+y_{s}{ }^{2}}\right) \exp \left[-i k \frac{x_{i} x_{l}+y_{i} y_{l}}{b}\right] \\
& \times \exp \left[i k \frac{x_{s}{ }^{2}+y_{s}{ }^{2}}{2 a}\right] \exp \left[-i k \frac{x_{l} x_{s}+y_{l} y_{s}}{a}\right],
\end{aligned}
$$

If we omit the term $\exp \left[i k\left(x_{s}^{2}+y_{s}^{2}\right) / 2 a\right]$ in Eq. (2), we get the Fraunhofer diffraction integral [21]. However, this is only valid for $a \gg\left(x_{s}^{2}+y_{s}^{2}\right)_{\max } / \lambda$. The secondary wave source has an effective radius of about $\gamma \lambda$ [22], so the Fraunhofer diffraction is only valid for $a \gg \gamma^{2} \lambda / 4 \pi^{2}$. In our previous work [19], we have shown that the distance from the radiator to the lens has a very weak influence on the image as long as the acceptance angle of the lens is much larger than $1 / \gamma$. Thus, we can generally adopt the Fraunhofer diffraction integral and omit the term $\exp \left[i k\left(x_{s}{ }^{2}+y_{s}{ }^{2}\right) / 2 a\right]$ in Eq. (2) while still maintaining 
the accuracy of the calculation. Because of the fact that the lens typically takes a circular shape, we can further perform the integration in polar coordinates where the following transformation is made: $x_{s}=R_{s} \cos \phi_{s}, y_{s}=R_{s} \sin \phi_{s}$, $x_{l}=R_{l} \cos \phi_{l}, \quad y_{l}=R_{l} \sin \phi_{l}, \quad x_{i}=R_{i} \cos \phi_{i}$, and $y_{i}=$ $R_{i} \sin \phi_{i}$. Note [23],

$$
\begin{aligned}
& \int_{0}^{2 \pi} d \varphi(\cos \varphi, \sin \varphi) \exp [i A \cos (\varphi-\phi)] \\
& \quad=2 \pi i(\cos \phi, \sin \phi) J_{1}(A) .
\end{aligned}
$$

After some mathematical manipulation, the field in the image plane is found to be

$$
\begin{aligned}
E_{x_{i}, y_{i}}^{i}= & \frac{4 \pi e \alpha}{\lambda^{2} M v}\left(\cos \phi_{i}, \sin \phi_{i}\right) \int d \theta \int d R_{s} \theta R_{s} K_{1}\left(\alpha R_{s}\right) \\
& \times J_{1}\left(k \theta R_{s}\right) J_{1}(\zeta \theta)
\end{aligned}
$$

where $M=b / a$ is the magnification factor, $\zeta=k R_{i} / M$, $\theta=R_{l} / a$ is integrated in the range $\left[0, \theta_{m}\right]$, and $\theta_{m}$ is the maximum acceptance angle of the lens.

The integration for $R_{s}$ in Eq. (3) should be performed from the inner radius to the outer radius of the radiator. However, carefully note the fact that the outer radius of the target is generally much larger than the effective radius of the pseudophoton disk for the optical component. Therefore, the integration for $R_{s}$ could be performed from $R_{0}$ to infinity, where $R_{0}$ is the radius of the aperture. Note [23],

$$
\begin{aligned}
\int_{0}^{r} x J_{n}(q x) K_{n}(p x) d x= & \frac{1}{q^{2}+p^{2}}\left[(q / p)^{n}+q r J_{n+1}(q r)\right. \\
& \left.\times K_{n}(p r)-p r J_{n}(q r) K_{n+1}(p r)\right], \\
\int_{0}^{\infty} x J_{1}(q x) K_{1}(p x) d x= & \frac{q}{q^{2} p+p^{3}},
\end{aligned}
$$

The field distribution in the image plane is further found to be

$$
\begin{aligned}
E_{x_{i}, y_{i}}^{i}= & \frac{2 e\left(\cos \phi_{i}, \sin \phi_{i}\right)}{\gamma \lambda M v} f\left(\theta_{m}, \gamma, \zeta, R_{0}\right) \\
f\left(\theta_{m}, \gamma, \zeta, R_{0}\right)= & \int d \theta \frac{J_{1}(\zeta \theta) \theta}{\theta^{2}+\gamma^{-2}}\left[\alpha R_{0} J_{1}\left(k R_{0} \theta\right) K_{2}\left(\alpha R_{0}\right)\right. \\
& \left.-k R_{0} \theta J_{2}\left(k R_{0} \theta\right) K_{1}\left(\alpha R_{0}\right)\right]
\end{aligned}
$$

Let us first consider a simple case when $R_{0}=0$, which is just the widely studied OTR case. Note,

$$
\begin{aligned}
\lim _{R_{0} \rightarrow 0} & {\left[\alpha R_{0} J_{1}\left(k R_{0} \theta\right) K_{2}\left(\alpha R_{0}\right)\right.} \\
& \left.-k R_{0} \theta J_{2}\left(k R_{0} \theta\right) K_{1}\left(\alpha R_{0}\right)\right]=\gamma \theta,
\end{aligned}
$$

One can find that Eq. (4) could be rewritten as

$$
E_{x_{i}, y_{i}}^{i}=\frac{2 e\left(\cos \phi_{i}, \sin \phi_{i}\right)}{\lambda M v} \int_{0}^{\theta_{m}} \frac{\theta^{2}}{\theta^{2}+\gamma^{-2}} J_{1}(\zeta \theta) d \theta,
$$

Equation (5) is in full agreement with that obtained in [16,19] which focuses on the resolution of imaging with OTR. Note that the field in Eq. (5) is obtained for a single electron. Thus, the intensity distribution in the image plane $I\left(x_{i}, y_{i}\right)=\frac{c}{4 \pi^{2}}\left(\left|E_{x_{i}}^{i}\right|^{2}+\left|E_{y_{i}}^{i}\right|^{2}\right)$ is just the image of a single electron and is therefore considered as the PSF of the OTR. The PSF of the OTR for various beam energies and lens acceptance angles is shown in Fig. 2.

From Fig. 2 we could see that the PSF is wider when the acceptance angle is smaller. This can be explained by the diffraction effect that the stronger one confines the photons with a diaphragm or a lens, the wider photons will extend after the passage. Figure 2 also shows that the PSF is almost independent of the energy. Actually, when the acceptance angle of the lens is much larger than $1 / \gamma$, Eq. (5) can be calculated in the following way with high precision:

$$
\begin{aligned}
E_{x_{i}, y_{i}}^{i} \approx & \frac{2 e\left(\cos \phi_{i}, \sin \phi_{i}\right)}{\lambda M v} \\
& \times\left[\int_{0}^{\infty} \frac{\theta^{2}}{\theta^{2}+\gamma^{-2}} J_{1}(\zeta \theta) d \theta-\int_{\theta_{m}}^{\infty} J_{1}(\zeta \theta) d \theta\right] \\
= & \frac{2 e\left(\cos \phi_{i}, \sin \phi_{i}\right)}{\lambda M v} \zeta^{-1}\left[\gamma^{-1} \zeta K_{1}\left(\gamma^{-1} \zeta\right)-J_{0}\left(\zeta \theta_{m}\right)\right],
\end{aligned}
$$

For a high-energy beam, we generally have $\gamma^{-1} \zeta \ll 1$, and Eq. (6a) can be further reduced to

$$
E_{x_{i}, y_{i}}^{i}=\frac{2 e\left(\cos \phi_{i}, \sin \phi_{i}\right)}{\lambda M v} \zeta^{-1}\left[1-J_{0}\left(\zeta \theta_{m}\right)\right],
$$

Equation (6b) implies that, for a high-energy beam and large acceptance angle, the PSF of the OTR is independent of energy, and that the characteristic angle relevant to the PSF is $\theta_{m}$ instead of $1 / \gamma$. This feature makes it attractive

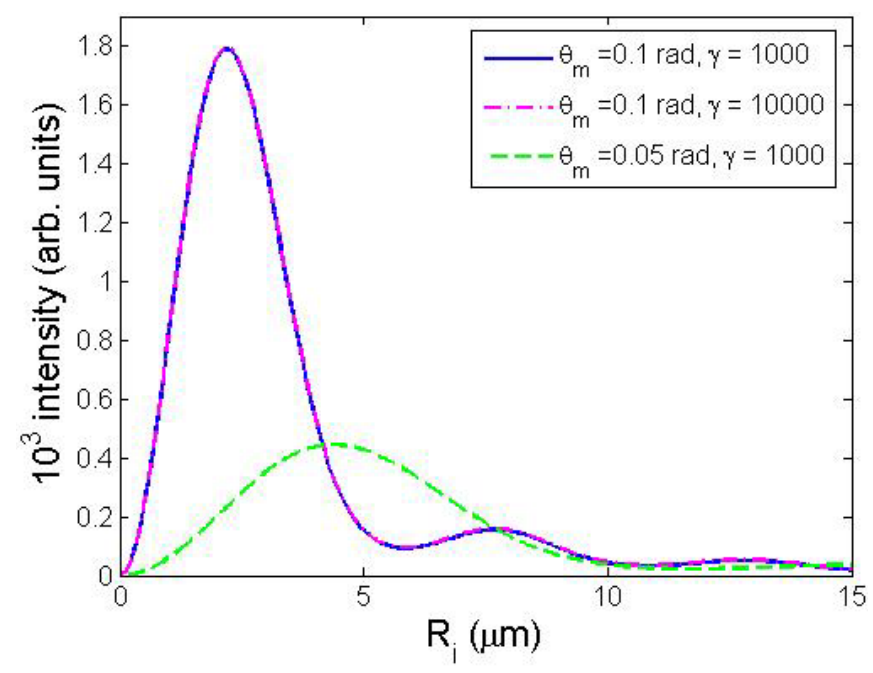

FIG. 2. (Color) PSF of the OTR for various beam energies and various lens acceptance angles. 
for high-energy beam diagnostics. The full width at half maximum (FWHM) of the PSF for the OTR is found to be approximately $M \lambda / \theta_{m}$, which is just 2 times larger than that for a point source. Further improvement in reducing the FWHM of the PSF can be achieved by increasing the acceptance angle of the imaging system and using the short wavelength component in doing the imaging.

Imagine that the OTR target is replaced with an ODR target while other things remain unchanged. In this scenario, it is natural to attribute the difference in imaging with ODR from that with OTR to the secondary wave source distribution on the target. For the OTR case, the source distribution is the same wherever the electron hits the target. However, for the ODR case, since the secondary wave source is only present on the target, its distribution depends on the radius of the aperture as well as the position at which the electron passes through it. As for imaging of high-energy electron beam with ODR, the condition $\alpha R_{0} \ll 1$ is typically satisfied and Eq. (4) can be approximately rewritten as

$$
\begin{aligned}
E_{x_{i}, y_{i}}^{i} \approx & \frac{2 e\left(\cos \phi_{i}, \sin \phi_{i}\right)}{\lambda M v} \\
& \times \int_{0}^{\theta_{m}} \frac{\theta^{2}}{\theta^{2}+\gamma^{-2}} J_{1}(\zeta \theta) J_{0}\left(k R_{0} \theta\right) d \theta,
\end{aligned}
$$

We see that when $R_{0}=0$, Eq. (7) is reduced to Eq. (5), which is just the OTR case. As the radius increases, the image evolves from that of OTR to ODR. Comparing Eq. (7) with Eq. (5), one could find that the image distribution of ODR is modulated by the function $J_{0}\left(k R_{0} \theta\right)$ as compared to that of the OTR. So, for the circular aperture with a very small radius that $2 \pi R_{0} \ll \lambda / \theta_{m}$, we have $J_{0}\left(k R_{0} \theta\right) \approx 1$, and there is no significant difference between the image generated by OTR and that by ODR. It is worth mentioning that the condition is much more stringent than that under which the angular distribution of the ODR approximately equals that of the OTR. The angular distribution of the ODR is modulated by the function $J_{0}\left(k R_{0} \gamma^{-1}\right)$ as compared to that of the OTR [22]. Thus, when the condition $2 \pi R_{0} \ll \gamma \lambda$ is satisfied, there is no big difference between the angular distribution of the ODR and that of the OTR. However, the condition for the ODR image to be close to the OTR image is $2 \pi R_{0} \ll \lambda / \theta_{m}$, which is far more stringent. The PSF of the ODR generated by circular aperture with various radii and various acceptance angles is numerically calculated and shown in Fig. 3, from which we could see that, for the same aperture, the difference between the ODR image and the OTR image is smaller when the acceptance angle is smaller, which is due to the fact that the condition $2 \pi R_{0} \ll \lambda / \theta_{m}$ is better satisfied when $\theta_{m}$ is smaller.

Figure 3 also indicates that the characteristic width of the PSF for the OTR (solid blue line) is approximately $\lambda / \theta_{m}$ instead of $\gamma \lambda$. This characteristic makes OTR applicable to the imaging of the very high-energy electron beam

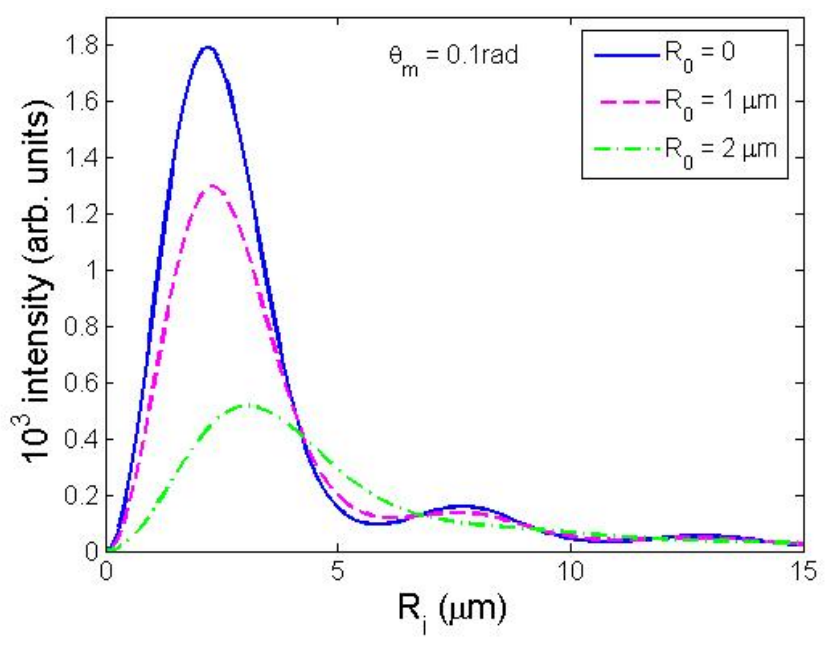

(a)

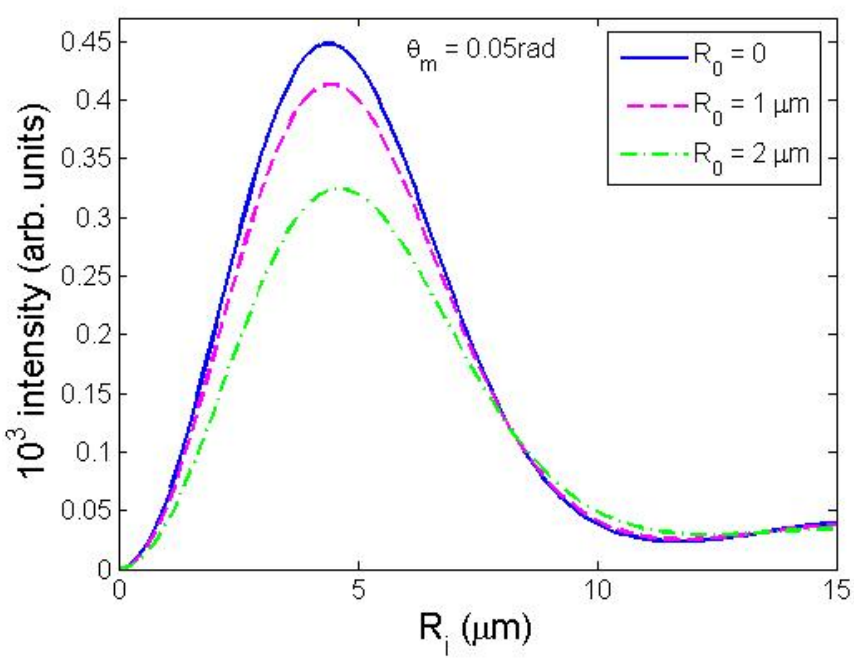

(b)

FIG. 3. (Color) PSF of the ODR generated from small radius circular aperture for various acceptance angles. (a) $\theta_{m}=$ $0.1 \mathrm{rad}$; (b) $\theta_{m}=0.05 \mathrm{rad}$. The parameters used are $\gamma=$ $10000, \lambda=0.5 \mu \mathrm{m}$, and $M=1$.

[2]. The image of a whole beam is found by convoluting the beam's distribution with the PSF. When the beam size is much larger than $\lambda / \theta_{m}$, then the difference between the real beam distribution and the image becomes negligible. Otherwise, the size of the image could be much larger than the real beam size, and one has to perform the deconvolution to retrieve the real beam profile [19].

Furthermore, one may see an interesting phenomenon that there is a considerable intensity in the region $R_{i}<R_{0}$ where the induced currents are not present. This could be explained as diffraction effects. Since the photons are confined to $\theta_{m}$, from the uncertainty principle, the photons can extend by about $\lambda / \theta_{m}$ into the region $R_{i}<R_{0}$.

For a practical case, the radius of the ODR target is typically in the range of a few hundred microns and we 
have $2 \pi R_{0} \gg \lambda / \theta_{m}$. Without using numerical integration, we can estimate the general shape of the PSF analytically. Most of the contribution in the integration is from $\theta>$ $1 / \gamma$, so that Eq. (7) may be approximately rewritten as

$$
E_{x_{i}, y_{i}}^{i} \approx \frac{2 e\left(\cos \phi_{i}, \sin \phi_{i}\right)}{\lambda M v} \int_{0}^{\theta_{m}} J_{1}(\zeta \theta) J_{0}\left(k R_{0} \theta\right) d \theta,
$$

When the condition $k R_{0} \theta_{m} \gg 1$ is satisfied, the integral above would have features very similar to that integrated from zero to infinity where we have [23]

$$
\int_{0}^{\infty} d \theta J_{1}(\zeta \theta) J_{0}\left(k R_{0} \theta\right)= \begin{cases}1 / \zeta & \zeta>k R_{0} \\ 1 /\left(2 k R_{0}\right) & \zeta=k R_{0} \\ 0 & \zeta<k R_{0}\end{cases}
$$

Equation (8) implies that for large radius aperture where $2 \pi R_{0} \gg \lambda / \theta_{m}$, the PSF should have no intensity in the region $R_{i}<M R_{0}$. The PSF should peak at $R_{i}=M R_{0}$ and decrease as $R_{i}$ further increases. Equation (8) also indicates that the PSF in the region $\zeta>k R_{0}$ is independent of the specific aperture radius and scales with proportion to $1 / \zeta$. This is in quantitative agreement with the results from numerical integration as shown in Fig. 4 where we see the PSF for circular aperture with various radii in the region $\zeta>k R_{0}$ overlap exactly.

The PSF of the ODR generated by circular aperture with radius $R_{0}=100 \mu \mathrm{m}$ and $R_{0}=200 \mu \mathrm{m}$ is numerically calculated from Eq. (7) and shown in Fig. 4. The peak value of PSF predicted by Eq. (8) for the $R_{0}=100 \mu \mathrm{m}$ aperture is 4 times larger than that of the $R_{0}=200 \mu \mathrm{m}$ aperture, which is also well reproduced in the numerical result. There is still one thing we would like to point out; when using Eq. (7), we have assumed the condition $\alpha R_{0} \ll 1$ to be satisfied. If the condition is not satisfied,

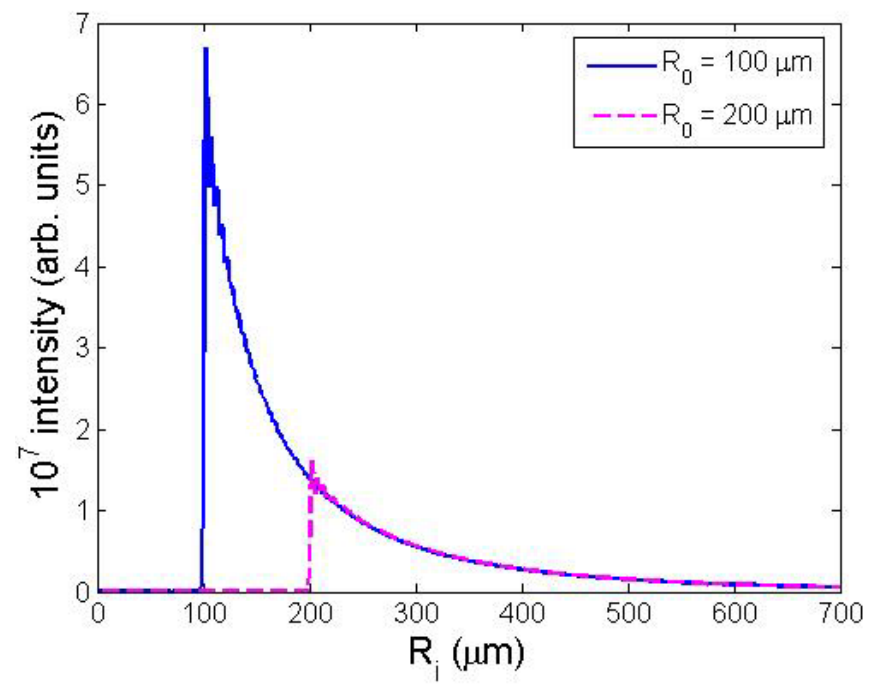

FIG. 4. (Color) PSF of the ODR generated from a large radius circular aperture. The parameters used are $\gamma=10000, \lambda=$ $0.5 \mu \mathrm{m}$, and $M=1$. then Eq. (4) should better describe the PSF than Eq. (7) does. The PSF when $\alpha R_{0}=0.628$ is calculated from the exact formula [Eq. (4)] and shown in Fig. 5. The result from the approximate formula [Eq. (7)] is also shown in the figure for comparison.

From Fig. 5 we could see, even for the condition $\alpha R_{0} \approx$ 1 , there is only a tiny difference between the result from Eq. (4) and that from Eq. (7). The role of the condition $\alpha R_{0} \ll 1$ is just to enable us to obtain a simple formula. The result from Eq. (7) slightly overestimates the PSF, because it neglects the second term of Eq. (4) in the brackets. Because of the same reason, the exact formula predicts no intensity in the region $R_{i}<M R_{0}$, while the result from the approximate formula takes some value. The difference is more significant when $\alpha R_{0}$ becomes larger than 1, and in that case it would be better to use Eq. (4) to study the PSF.

From Fig. 4 we see that, as compared to the OTR case, the PSF of the ODR shows some special feature that the intensity only exists in the outer region whose radius exactly equals that of the circular aperture on the target (generally the value equals $M R_{0}$ ). This feature makes the ODR image differ greatly from the real beam profile. However, this does not mean that it is impossible to use the ODR to image beam profile. Since the PSF is calculable, in principle, the beam's profile can be obtained by performing deconvolution of the measured image with the PSF. Like all inverse problems, this restoration would not be trivial. The most difficult job for this may be that the PSF is space variant because different electrons will have different offsets with respect to the aperture center and the PSF is different too. In order to perform the deconvolution, we need to study the case when the electron passes through the aperture with some offset to the center. An approximate formula describing the angular distribution of DR when the

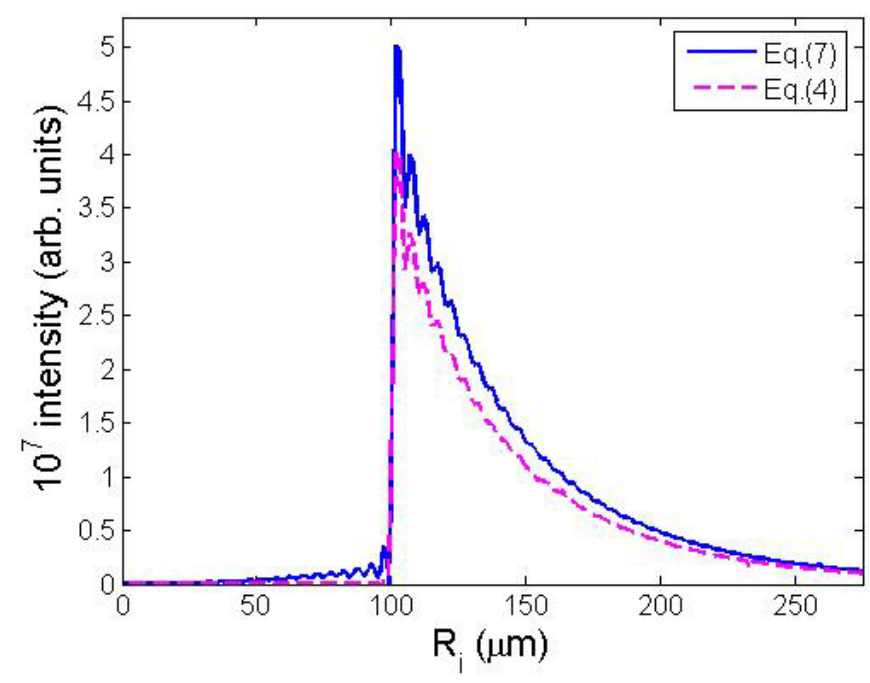

FIG. 5. (Color) Comparison of PSF of ODR obtained from Eq. (4) and that from Eq. (7). The parameters used are $\gamma=$ 2000, $\lambda=0.5 \mu \mathrm{m}$, and $M=1$. 
electron passes through a circular aperture with a nonzero offset to the aperture center has been derived [see Eq. (31.15) in Ref. [20]] under the assumption that the offset is much smaller than the aperture radius and $\alpha R_{0} \ll 1$. This formula has been further used by the authors in [24,25] to study the DR angular distribution of a real beam when the beam center passes through the circular aperture. However, one should note that the formula [Eq. (31.15) in Ref. [20] ] only contains the first two terms of the infinite series, which is only justified for the case when $\alpha R_{0} \ll 1$. For a more general and interesting case when $\alpha R_{0} \sim 1$, the investigations on the PSF should rely on the numerical method and could be quite complex. Here, we omitted this study and, alternatively, we will study the imaging with ODR from a rectangular slit and semi-infinite plane for which the beam's position effect on PSF is much easier to handle.

\section{IMAGING WITH ODR FROM A RECTANGULAR SLIT}

Consider a similar geometry as that in Fig. 1, wherein the circular aperture is replaced with a rectangular slit which has a width of $t$ in the vertical direction. The slit is assumed to be infinitely large in the horizontal direction and the electron passes through the slit at a distance of $t_{1}$ and $t_{2}$ with respect to the lower and upper edge of the slit, respectively. We found out that for this case it is more convenient to rewrite Eq. (1) in the following form [20]:

$$
\begin{aligned}
E_{x_{s}, y_{s}}^{s}= & -\frac{i e}{2 \pi^{2} v} \int d q_{x} d q_{y} \frac{q_{x, y}}{q_{x}^{2}+q_{y}^{2}+\alpha^{2}} \\
& \times \exp \left[i\left(q_{x} x_{s}+q_{y} y_{s}\right)\right],
\end{aligned}
$$

Under the assumption of Fraunhofer diffraction and after similar procedures, the field in the image plane for DR generated by a rectangular slit is found to be

$E_{x_{i}, y_{i}}^{i}=\frac{i e}{2 \pi^{2} \lambda^{2} a b v} \int d x_{l} d y_{l} \int d x_{s} d y_{s} \int d q_{x} d q_{y} \frac{q_{x, y} \exp \left[i\left(q_{x} x_{s}+q_{y} y_{s}\right)\right]}{q_{x}^{2}+q_{y}^{2}+\alpha^{2}} \exp \left[-i k \frac{x_{i} x_{l}+y_{i} y_{l}}{b}\right] \exp \left[-i k \frac{x_{l} x_{s}+y_{l} y_{s}}{a}\right]$

After some mathematical manipulation, Eq. (9) can be reduced to (see the Appendix)

$$
\begin{aligned}
E_{x_{i}}^{i}= & \frac{i e}{2 \pi \lambda M v} \int d \theta d \phi_{l} \frac{\theta^{2} \cos \phi_{l}}{\sqrt{\left(\theta \cos \phi_{l}\right)^{2}+\gamma^{-2}}} \exp \left[-i k \frac{x_{i} \theta \cos \phi_{l}+y_{i} \theta \sin \phi_{l}}{M}\right] \\
& \times\left[\frac{e^{-k t_{1}}\left(\sqrt{\left(\theta \cos \phi_{l}\right)^{2}+\gamma^{-2}}-i \theta \sin \phi_{l}\right)}{\sqrt{\left(\theta \cos \phi_{l}\right)^{2}+\gamma^{-2}}-i \theta \sin \phi_{l}}+\frac{e^{-k t_{2}\left(\sqrt{\left(\theta \cos \phi_{l}\right)^{2}+\gamma^{-2}}+i \theta \sin \phi_{l}\right)}}{\sqrt{\left(\theta \cos \phi_{l}\right)^{2}+\gamma^{-2}}+i \theta \sin \phi_{l}}\right], \\
E_{y_{i}}^{i}= & \frac{i e}{2 \pi \lambda M v} \int d \theta d \phi_{l} \theta \exp \left[-i k \frac{x_{i} \theta \cos \phi_{l}+y_{i} \theta \sin \phi_{l}}{M}\right] \\
& \times\left[\frac{e^{-k t_{1}\left(\sqrt{\left(\theta \cos \phi_{l}\right)^{2}+\gamma^{-2}}-i \theta \sin \phi_{l}\right)}}{\sqrt{\left(\theta \cos \phi_{l}\right)^{2}+\gamma^{-2}}-i \theta \sin \phi_{l}}-\frac{e^{-k t_{2}\left(\sqrt{\left(\theta \cos \phi_{l}\right)^{2}+\gamma^{-2}}+i \theta \sin \phi_{l}\right)}}{\sqrt{\left(\theta \cos \phi_{l}\right)^{2}+\gamma^{-2}}+i \theta \sin \phi_{l}}\right],
\end{aligned}
$$

Note that when the slit width is zero, we have $t_{1}=t_{2}=$ 0 and Eq. (10) exactly reduces to Eq. (5), which indicates the consistency in our calculation. When the electron center passes through the slit with the parameters $t_{1}=$ $t_{2}=100 \mu \mathrm{m}$, the images formed with horizontal polarization component and vertical polarization component are calculated from Eq. (10) and shown in Figs. 6(a) and 6(b), respectively. Hereafter in this paper, unless otherwise mentioned, we will use the parameters $\gamma=2500, \theta_{m}=0.1$, $\lambda=0.5 \mu \mathrm{m}$, and $M=1$.

To emphasize the shape of the PSF, the intensity of each PSF in Fig. 6 is normalized to their specific peak value and thus are not scaled with each other. The peak intensity of the vertical component is about 7 times larger than that of the horizontal component. The relative value of the vertical component to the horizontal component depends on the beam energy, the slit width, and the wavelength. For the OTR case where the slit width is zero, they have equal peak value. As the value $t / \gamma \lambda$ increases, so does the ratio of the peak value of the vertical component to that of the horizontal component. Here we will concentrate on imaging with the vertical component taking advantage of its relatively large intensity.

The 1D distribution of the image formed with the vertical component for various slit widths when $x_{i}=0$ is shown in Fig. 7.

From Fig. 7 we see that, when the electron center passes through the slit, the image is symmetric. Other things being equal, the intensity of the image is larger for a narrower slit. However, when the electron passes through the slit with nonzero offset to the slit center, the image should have an asymmetric shape. Consider a slit whose width is 


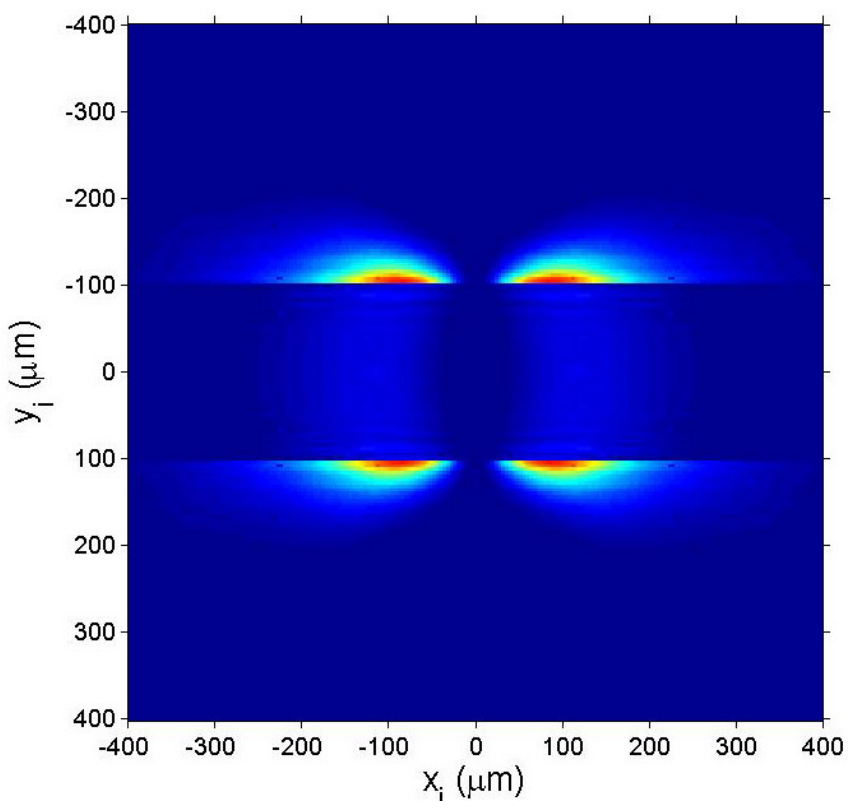

(a)

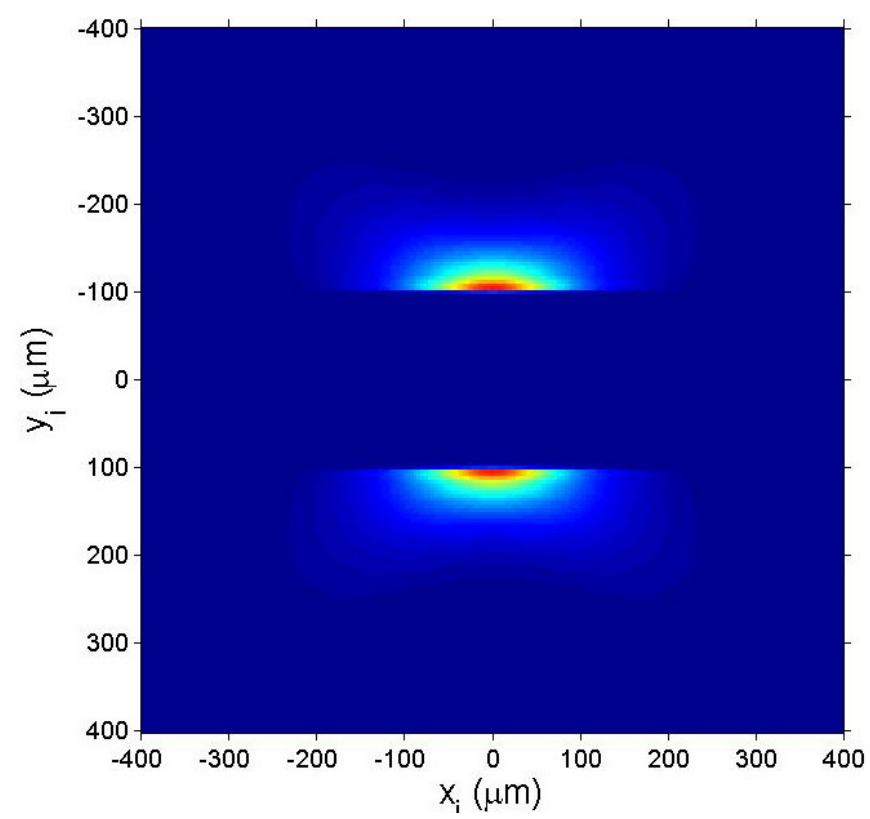

(b)

FIG. 6. (Color) PSF of the ODR from rectangular slit with horizontal component (a) and with vertical component (b).

$100 \mu \mathrm{m}$, the image formed with the ODR when the electron passes through the slit with $t_{1}=30 \mu \mathrm{m}$ and $t_{2}=$ $70 \mu \mathrm{m}$ is shown in Fig. 8.

From Fig. 8 we could see that, when the electron passes through the slit with nonzero offset to the slit center, the image becomes asymmetric in both shape and intensity. The ratio of the intensity for the two peaks depends on the offset of the electron. Thus, we may use the ODR image to monitor the beam position in the direction perpendicular to the slit edge. The ratio of the two peak intensities as a

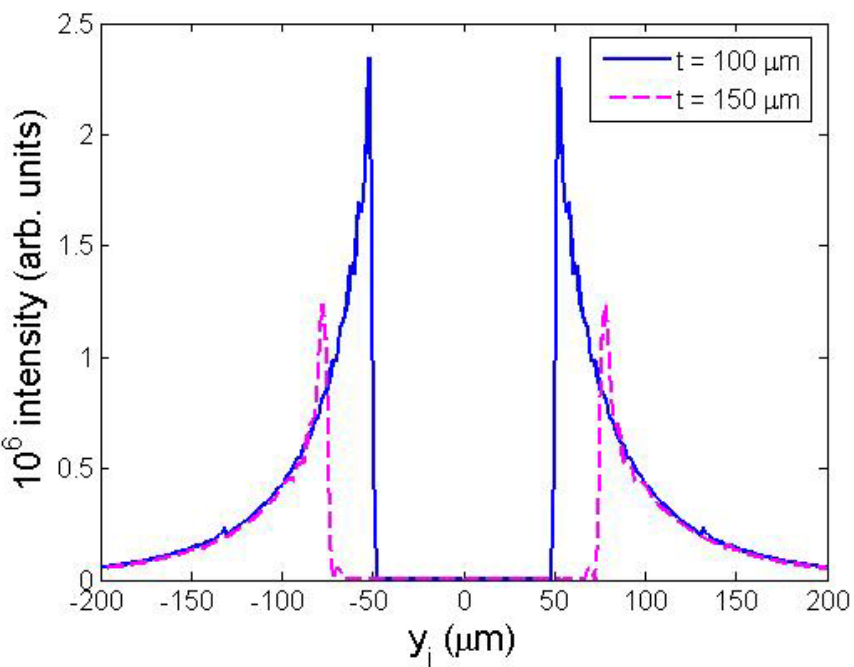

FIG. 7. (Color) 1D image formed with the ODR when a single electron center passes through a rectangular slit with various widths.

function of the offset with respect to the slit center is calculated and shown in Fig. 9.

From Fig. 9 we see that the beam position can be inferred from the image and the resolution could be as high as a few microns. The resolution may meet the diagnosis requirement of ILC in the main linac [3]. This method could be applied to real-time monitor the beam position variation in the long undulator for the XFEL, which is very helpful to preserve overlapping between the self-amplified x-ray and the driving electron beam.

For the proposed ILC beam [3] or the beam from the high performance damping ring [9], since the vertical beam size is so small that for simplicity the beam distribution may be approximately written as

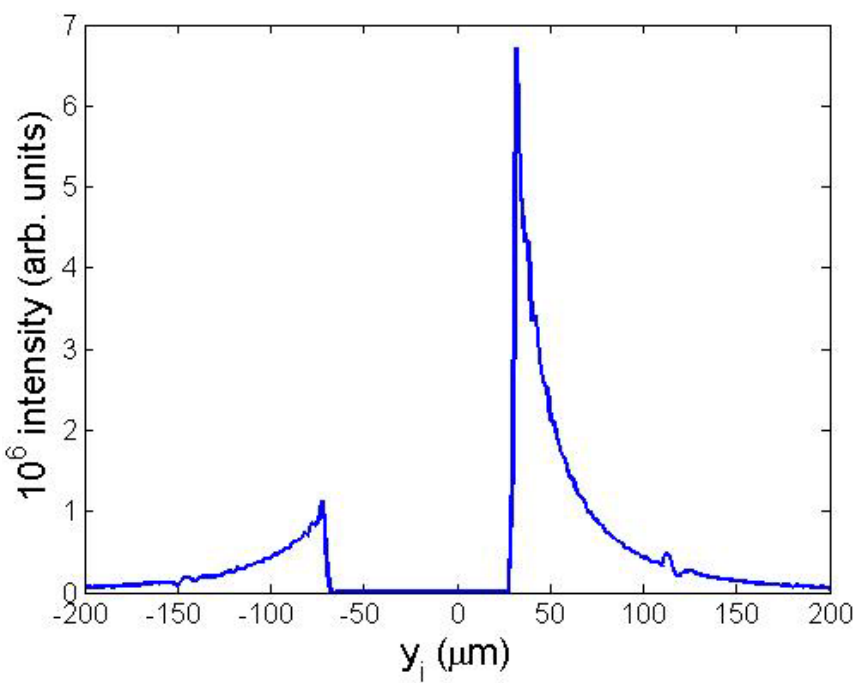

FIG. 8. (Color) 1D image formed with the ODR when a single electron passes through a rectangular slit with $20 \mu \mathrm{m}$ offset to the slit center. $x_{i}=0$. 


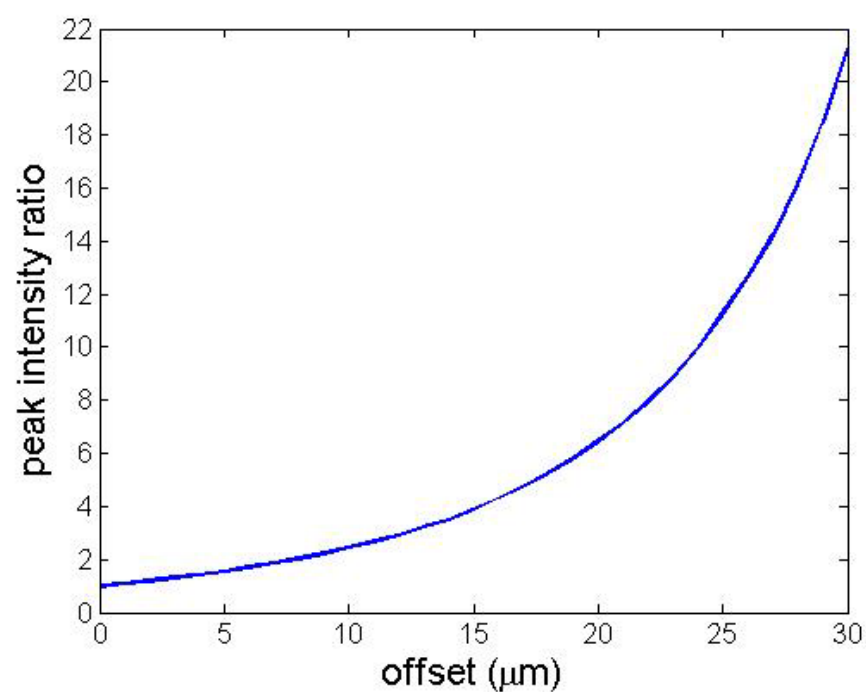

FIG. 9. (Color) Sensitivity of the peak intensity ratio to the beam offset.

$$
s(x, y) \approx \frac{1}{\sqrt{2 \pi} \sigma_{x}} \exp \left(-x^{2} / 2 \sigma_{x}^{2}\right) \delta(y),
$$

where $\delta(y)$ is the Dirac delta function. The description of the delta function could simplify the convolution process. The image for the whole beam is found by convoluting the beam's distribution with the PSF. The image with a vertical component for the beam with $\sigma_{x}=100 \mu \mathrm{m}$ is shown in Fig. 10(a) when the beam center passes through the slit with width $t=200 \mu \mathrm{m}$.

The projected 1D profile $I_{p}\left(x_{i}\right)$ is defined as $I_{p}\left(x_{i}\right)=$ $\int I\left(x_{i}, y_{i}\right) d y_{i}$, where $I\left(x_{i}, y_{i}\right)$ is the intensity distribution of the 2D image. The projected 1D profile of the PSF, the beam, the image, and the restored beam from deconvolution is shown in Fig. 10(b). From Fig. 10(b) we could see that the image size is about 50\% larger than the real beam size. The real beam profile could be obtained after deconvoluting the image with the PSF. A brief discussion about the procedures and accuracy of deconvolution with various algorithms could be found in our previous work [19]. For effective use of space, we just present the results here. With the well-known Lucy-Richardson algorithm [19,26,27], the restored beam profile is obtained and shown in Fig. 10(b) with the red circle. From Fig. 10 we can conclude that, even though the ODR image differs greatly from the real beam profile, the real beam profile could be restored with excellent accuracy after deconvolution.

In a real experiment, noise is always present. Deconvolution of a noisy image is generally affected by noise amplification. This is caused by the fact that the image and real beam distribution are both generally band limited and their amplitude would decrease as the spatial frequency increases. However, the noise does not behave this way, and considering the low photon yield of the ODR, the noise problem may be severe. Random numbers (noise) whose elements are normally distributed with a mean of 0

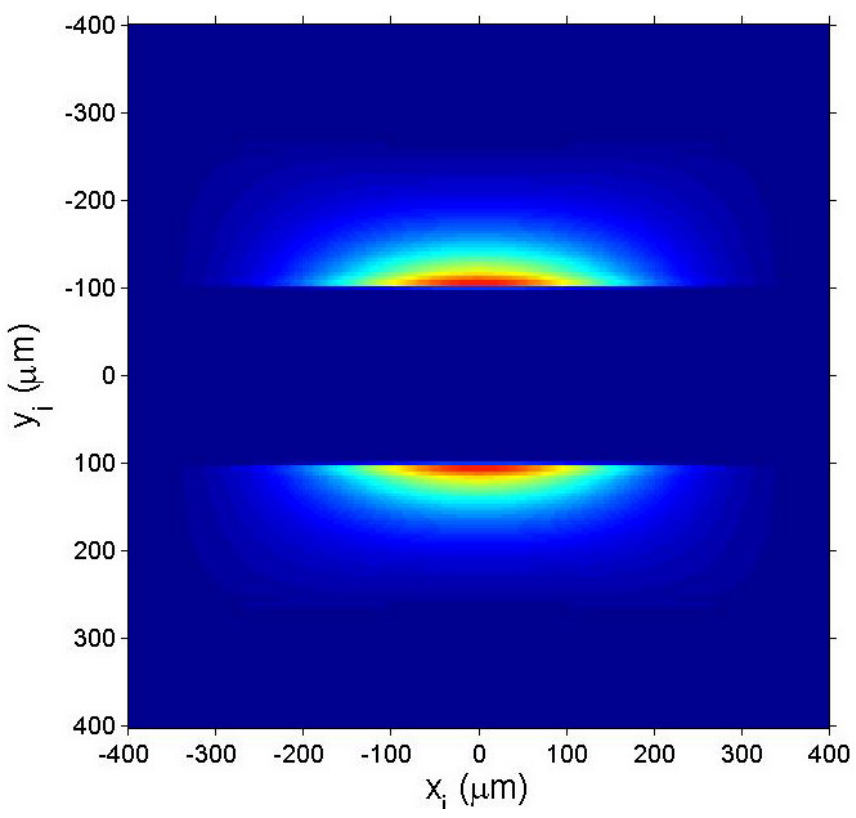

(a)

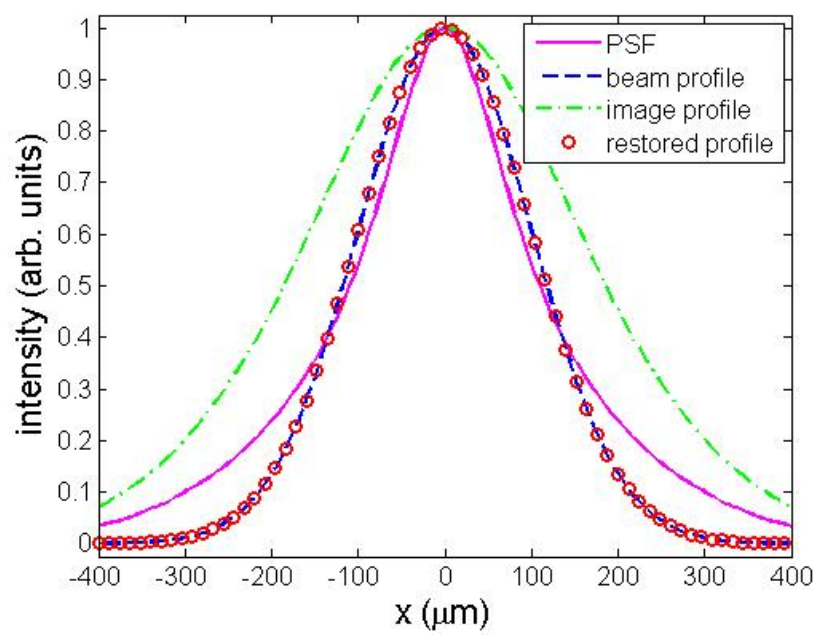

(b)

FIG. 10. (Color) The ODR image for a beam with $\sigma_{x}=$ $100 \mu \mathrm{m}$ (a); the projected 1D profile of the PSF, the beam, the image, and the restored beam from deconvolution (b).

and standard deviation of 1 are first multiplied by 0.05 times the peak intensity of the image and then added to the noise free image as in Fig. 10(a). The resulted noisy image is shown in Fig. 11(a).

The projected profile of the ODR image is quite noisy as shown by the dashed green line in Fig. 11(b). Fortunately, with the Richardson-Lucy algorithm, the restored beam distribution is still in satisfactory agreement with the real beam profile, except for some tiny difference in the peak intensity region. This is a strong confirmation of the feasibility of predicting the beam profile from the noisy ODR image using the deconvolution method. 


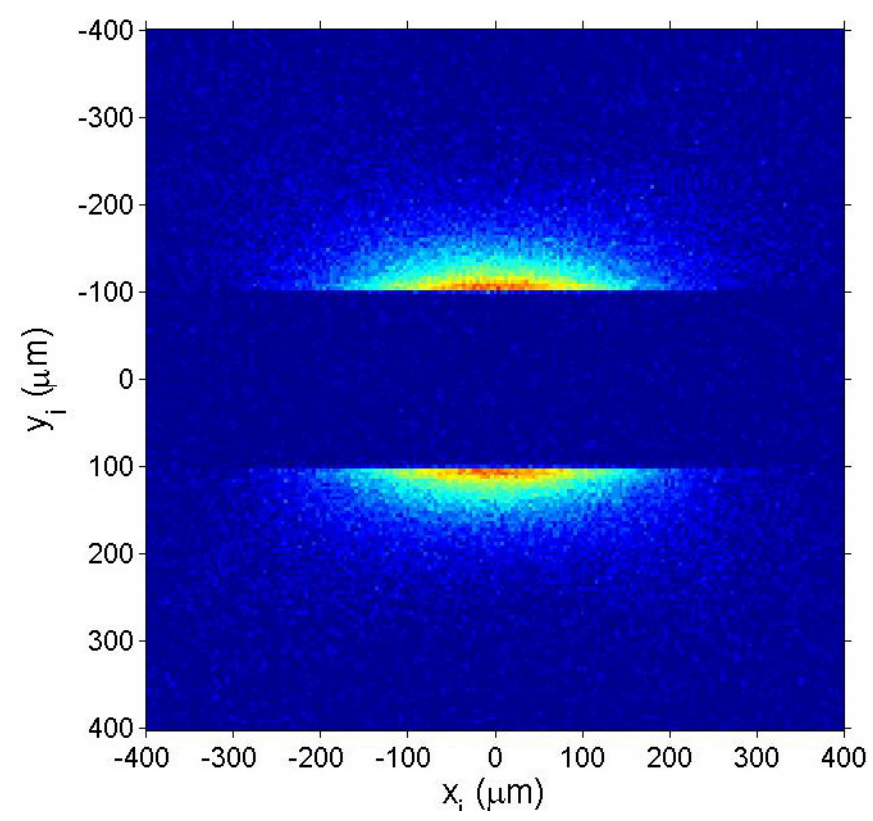

(a)

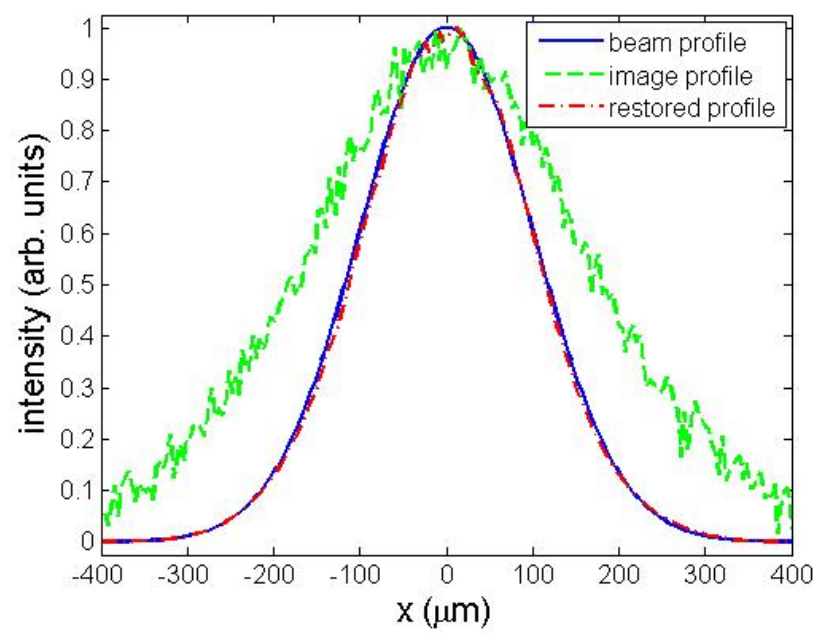

(b)

FIG. 11. (Color) The ODR image with noise for a beam with $\sigma_{x}=100 \mu \mathrm{m}$ (a); the projected 1D profile of the beam, the noisy image, and the restored beam distribution (b).

The projected distribution of the PSF is non-Gaussian as shown by the solid magenta line in Fig. 10(b). For fast estimation of the size difference in image formed with OTR and ODR, we may roughly use the Gaussian distribution as a first approximation. Recall the fact that the convolution of two Gaussian functions is also Gaussian with standard deviation being the square root of the sum of the square of the standard deviation for each Gaussian distribution; we have

$$
\sigma_{i}=\sqrt{\sigma_{b}^{2}+\sigma_{p}^{2}}
$$

where $\sigma_{i}, \sigma_{b}$, and $\sigma_{p}$ are the rms size of the image, the beam, and the PSF, respectively. The image formed with OTR is generally taken to be the same as the real beam profile when the beam size is much larger than $\lambda / \theta_{m}$, so we can write the ratio of the beam size formed with ODR to that formed with OTR as $\sqrt{1+\sigma_{p}^{2} / \sigma_{b}^{2}}$ which indicates that the ratio is always larger than 1 and decreases as the beam size increases. This is in good agreement with the experimental results [11-14]. For example, it is shown in Fig. 9 of Ref. [14] that the ratio is maximal when the beam size is minimal and decreases as the beam size increases.

\section{IMAGING WITH ODR FROM A SEMI-INFINITE PLANE}

Equation (10) indicates that the ODR field for a rectangular slit could be considered as a superposition of that from the lower plane and the upper plane. By taking $t_{1} \rightarrow$ $\infty$ or $t_{2} \rightarrow \infty$ in Eq. (10), the contribution from the lower plane or from the upper plane approaches zero, and the corresponding field for a semi-infinite plane is obtained. Consider a semi-infinite plane which is assumed to be infinitely large in the horizontal direction and the electron passes beneath the target with distance $t_{2}$ to the lower edge of the plane. Take the vertical component as an example, the PSF when $t_{2}=100 \mu \mathrm{m}$ is calculated and shown in Fig. 12.

A comparison between Fig. 6(b) and Fig. 12 implies that the PSF of the ODR from a semi-infinite plane approximately equals half of that from the rectangular slit. Mathematically, this is due to the fact that each term in the brackets of Eq. (10) only contributes to some local region in the image plane. Equation (10) indicates that the

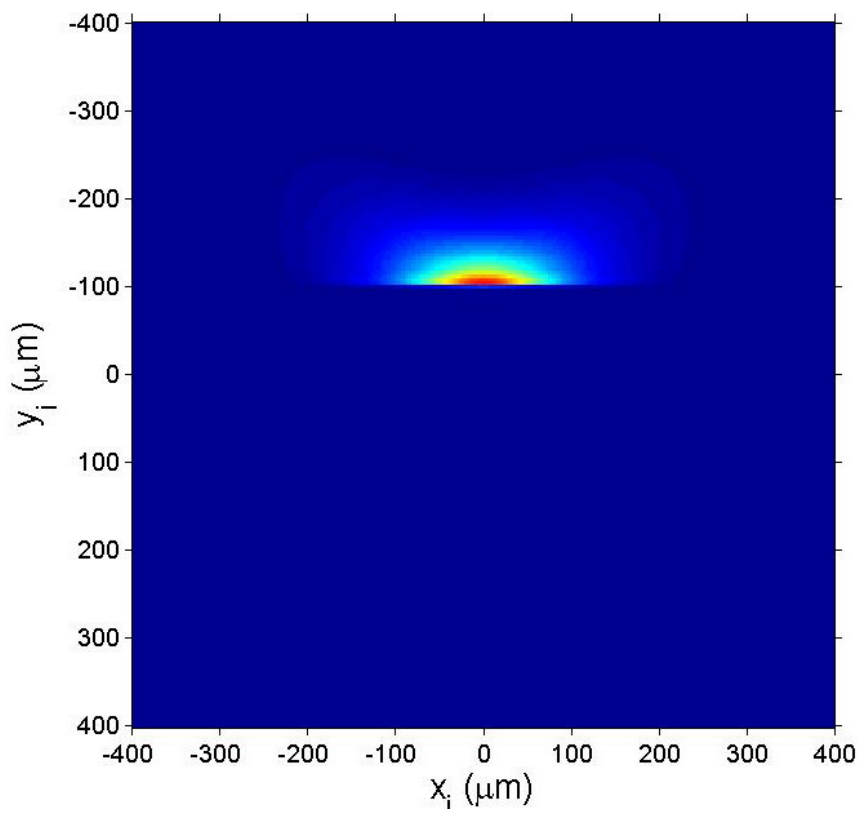

FIG. 12. (Color) PSF of ODR from a semi-infinite plane. 
phase difference between the field from the upper plane and that from the lower plane is $2 \pi t \theta \sin \phi_{l} / \lambda$. Since the interference effect is only important when the phase difference is smaller than $1 \mathrm{rad}$, we conclude that, as long as the slit width is larger than $\lambda / \theta_{m}$, the interference between the fields from the upper plane and lower plane could be neglected. Since it is only the extreme case of ODR from a rectangular slit, the deconvolution method described in the section above should be applicable to this geometry too.

From Fig. 12 we also see that the PSF peaks at $y_{i}=$ $-100 \mu \mathrm{m}$ (more generally when $y_{i}=-M t_{2}$ ) and smoothly deceases in the region $y_{i}<-100 \mu \mathrm{m}$, while no considerable intensity is present in the region $y_{i}>$ $-100 \mu \mathrm{m}$. To see how this happens, the induced currents distribution on the target is shown in Fig. 13.

A comparison between Fig. 12 and Fig. 13 indicates that the position of the peak intensity of the PSF depends on that of the induced currents. As long as the electron passes beneath the target, the induced currents are always maximal at the target edge. Therefore, the peak intensity position of the PSF is only dependent on the position of the target edge. As for the electron beam with some vertical dimension, the peak intensity position of the image for the whole beam should be independent of the beam's vertical position and vertical size. Figure 14 shows the 1D projected distribution of the induced currents on the target when beam passes beneath the semi-infinite plane with various distances and various vertical sizes.

The distance $t_{0}$ is measured from the beam centroid to the target edge. From Fig. 14 we could see that, while the intensity of the induced currents does depend on the beam's position and size, the position of the peak intensity is only dependent on the position of the target edge. Thus,

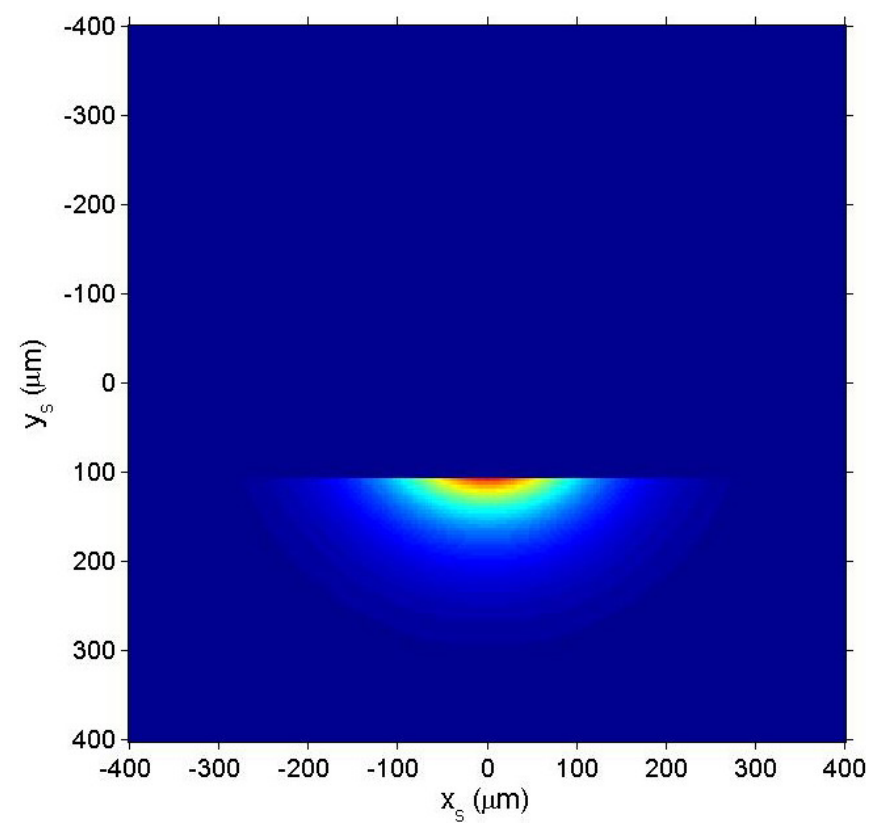

FIG. 13. (Color) Induced currents distribution on the target.

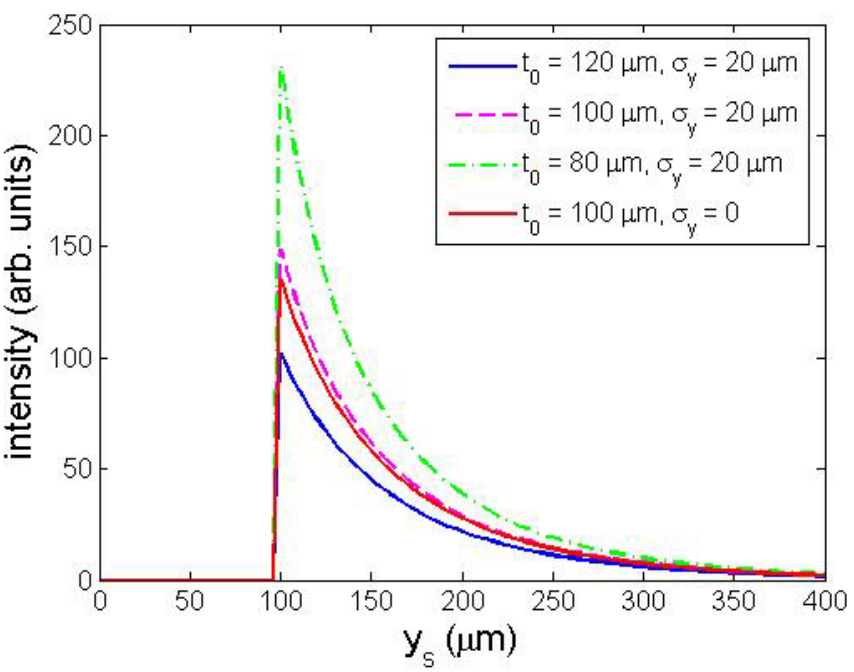

FIG. 14. (Color) 1D projected distribution of the induced currents on the target for a beam.

unlike the case in the direction parallel to the edge for which the position of the image directly represents the position of the beam, one cannot use the position of the image to predict the beam position in the direction perpendicular to the edge. This is because the peak intensity position in this direction only reflects the position of the target edge. Alternatively, as mentioned in Sec. III, one could use the rectangular slit to predict the beam position in this direction via the peak intensity ratio as shown in Fig. 9.

Furthermore, a comparison between the dashed magenta and solid red lines in Fig. 14 would imply that the induced currents distribution is only slightly dependent on beam size, and so is the ODR image. Based on this, we predict that the image for a beam in the vertical direction should be very similar to that of a single electron, because the induced currents for both are very similar. The ODR image of a beam should have a sharp peak in the vertical direction, just as sharp as that for a single electron (for example, see Fig. 7). This is in good agreement with the experimental results reported in [11-14] where it is shown that the peak of the image obtained with ODR is much narrower than that obtained with OTR in the vertical direction. The physical picture of this phenomenon is that the image directly reproduces the distribution of the induced currents from which the ODR photons are generated. We indeed monitor the beam via monitoring the induced currents. For a whole beam, the induced currents distribution in the vertical direction on the semi-infinite plane is similar to that for a single electron. Therefore, the ODR image in the direction perpendicular to the plane edge does not show similarity with the beam distribution.

If the ODR target is shifted vertically, we can expect that both the peak intensity position and the intensity of the image will change, and that the peak intensity position should change linearly with that of the ODR target. This 
is in good agreement with that observed in [11-14]. On the contrary, when the beam is shifted vertically while the ODR target remains unmoved, the position of the image will not change because the induced currents remain unmoved and only the intensity will change. Thus, we conclude using the image from the semi-infinite plane to monitor beam position variation in the direction parallel to the plane edge is straightforward. However, it cannot be used to monitor beam position variation in the direction perpendicular to the plane edge. A possible way to monitor the beam position in such a direction is to employ the rectangular slit geometry. As shown in Fig. 9, the peak intensity ratio of the image would give the beam position information. Thus, it seems that the rectangular slit geometry is a better candidate for monitoring beam position variation, because it can monitor the beam position in both the horizontal and the vertical directions. Recent experimental results [11-14] are encouraging in terms of the resolution of monitoring beam position variation. Nevertheless, further experiments are needed to validate other theoretical predictions made in the sections above.

\section{CONCLUSIONS AND DISCUSSIONS}

There is growing interest in developing nonintercepting methods to characterize beam parameters that are motivated by the challenge of ILC and XFELs where the high intensity beam excludes the use of the intercepting method. Moreover, real-time monitoring of the beam parameters is highly preferable. In this paper we investigated the possibility of using ODR to image beam profile. The image of a single electron measured with the ODR is studied from first principle. We found that the image formed with ODR from a rectangular slit could be used to monitor the beam's position in both the horizontal and the vertical directions. If deconvolution is performed, the image formed with ODR from a rectangular slit and semi-infinite plane could be used to determine the beam profile.
Up to now, only some preliminary experimental results have been reported where the beam size is quite large and ODR is generated from a semi-infinite plane. To draw a sound conclusion on whether or not ODR imaging is really applicable to the beam characterizations for the proposed ILC and XFELs, further investigations with small size beam should be performed. KEK-ATF, FFTB in SLAC, and other high performance high-energy accelerators are good candidates for such kinds of experiments. As an example, we used the typical parameters of KEK-ATF in most of the calculations. The perturbations to the beam during the use of ODR also need to be studied. Since the backward DR propagates in the direction perpendicular to the trajectory, it is expected that a transverse kick would be added to the beam as a result of momentum conservation $[28,29]$. Furthermore, when the electron beam passes through the DR target, electrons with different transverse positions will lose different energies due to the different impact factors. This $r$-dependent energy loss would cause a $z$-dependent kick due to the Panofsky-Wenzel theorem and would cause projected emittance growth. These issues should be investigated in future work to fully justify if ODR is practically suitable for real-time beam diagnosis.

\section{ACKNOWLEDGMENTS}

This work was supported by the Chinese National Foundation of Natural Sciences under Contract No. 10475047.

\section{APPENDIX}

Considering the Fraunhofer case, starting from Eq. (9), the integration for $x_{s}$ is direct and yields $2 \pi \delta\left(q_{x}-k x_{l} / a\right)$. The integration for $y_{s}$ should be performed from $-\infty$ to $-t_{1}$ and from $t_{2}$ to $\infty$. After this procedure, Eq. (9) is reduced to

$$
\begin{aligned}
E_{x_{i}, y_{i}}^{i} & =\frac{2 i e}{\lambda^{2} a b v} \int d x_{l} d y_{l} \frac{k\left(x_{l}, y_{l}\right) / a}{\left(k x_{l} / a\right)^{2}+\left(k y_{l} / a\right)^{2}+\alpha^{2}} \exp \left[-i k \frac{x_{i} x_{l}+y_{i} y_{l}}{b}\right]-\left[I_{1}-I_{2}\right], \\
I_{1} & =\frac{i e}{\pi \lambda^{2} a b v} \int d x_{l} d y_{l} \exp \left[-i k \frac{x_{i} x_{l}+y_{i} y_{l}}{b}\right] \int d q_{x} d q_{y} \frac{\delta\left(q_{x}-k x_{l} / a\right)}{i y_{s}\left(q_{y}-k y_{l} / a\right)} \frac{q_{x, y}}{q_{x}^{2}+q_{y}{ }^{2}+\alpha^{2}} \exp \left[i t_{2}\left(q_{y}-k y_{l} / a\right)\right], \\
I_{2} & =\frac{i e}{\pi \lambda^{2} a b v} \int d x_{l} d y_{l} \exp \left[-i k \frac{x_{i} x_{l}+y_{i} y_{l}}{b}\right] \int d q_{x} d q_{y} \frac{\delta\left(q_{x}-k x_{l} / a\right)}{i y_{s}\left(q_{y}-k y_{l} / a\right)} \frac{q_{x, y}}{q_{x}^{2}+q_{y}{ }^{2}+\alpha^{2}} \exp \left[-i t_{1}\left(q_{y}-k y_{l} / a\right)\right],
\end{aligned}
$$

An integration similar to Eqs. (A1a) and (A1b) had been performed in Ref. [6]. Here we take Eq. (A1a) as an example, the integral for $q_{x}$ is straightforward, and for $q_{y}$ there are 3 poles at $q_{y}= \pm \sqrt{\left(k x_{l} / a\right)^{2}+\alpha^{2}} i$ and $q_{y}=k y_{l} / a$. The integral can be performed directly with proper contour as shown in the appendix of Ref. [6]. After this procedure the radiation field is found to be 


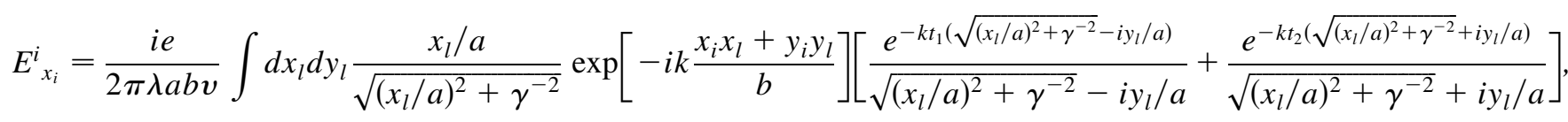

$E_{y_{i}}^{i}=\frac{e}{2 \pi \lambda a b v} \int d x_{l} d y_{l} \exp \left[-i k \frac{x_{i} x_{l}+y_{i} y_{l}}{b}\right]\left[\frac{e^{-k t_{1}\left(\sqrt{\left(x_{l} / a\right)^{2}+\gamma^{-2}}-i y_{l} / a\right)}}{\sqrt{\left(x_{l} / a\right)^{2}+\gamma^{-2}}-i y_{l} / a}-\frac{e^{-k t_{2}\left(\sqrt{\left(x_{l} / a\right)^{2}+\gamma^{-2}}+i y_{l} / a\right)}}{\sqrt{\left(x_{l} / a\right)^{2}+\gamma^{-2}}+i y_{l} / a}\right]$

After transforming to cylindrical coordinates, we finally get Eq. (10).

[1] C. Bal, E. Bravin, E. Chevallay, T. Lefèvre, and G. Suberlucq, Proceedings of the DIPAC03, Mainz, 2003, p. 95.

[2] P. Catravas, W. P. Leemans, E. Esarey, M. Zolotorev, D. Whittum, R. Iverson, M. Hogan, and D. Walz, Proceedings of the PAC99, New York, 1999, p. 2111.

[3] P. Tenenbaum, AIP Conf. Proc. 868, 138 (2006).

[4] A.P. Potylitsyn, Nucl. Instrum. Methods Phys. Res., Sect. B 145, 169 (1998).

[5] M. Castellano, Nucl. Instrum. Methods Phys. Res., Sect. A 394, 275 (1997).

[6] R. B. Fiorito and D. W. Rule, Nucl. Instrum. Methods Phys. Res., Sect. B 173, 67 (2001).

[7] J. Urakawa, H. Hayano, K. Kubo, S. Kuroda, N. Terunuma, M. Kuriki, T. Okugi, T. Naito, S. Araki, A. Potylitsyn, G. Naumenko, P. Karataev, N. Potylitsyna, I. Vnukov, T. Hirose, R. Hamatsu, T. Muto, M. Ikezawa, and Y. Shibata, Nucl. Instrum. Methods Phys. Res., Sect. A 472, 309 (2001).

[8] N. Potylitsina-Kube and X. Artru, Nucl. Instrum. Methods Phys. Res., Sect. B 201, 172 (2003).

[9] Pavel Karataev, Sakae Araki, Ryosuke Hamatsu, Hitoshi Hayano, Toshiya Muto, Gennady Naumenko, Alexander Potylitsyn, Nobuhiro Terunuma, and Junji Urakawa, Phys. Rev. Lett. 93, 244802 (2004).

[10] Dao Xiang and Wen-Hui Huang, Nucl. Instrum. Methods Phys. Res., Sect. B 254, 165 (2007).

[11] A. H. Lumpkin, W. J. Berg, N. S. Sereno, D. W. Rule, B. X. Yang, and C. Y. Yao, Proceedings of FEL 2005, p. 604.

[12] A. H. Lumpkin, N. S. Sereno , W. J. Berg, D. W. Rule, B.X. Yang, and C. Y. Yao, Proceedings of FEL 2006, p. 710 .

[13] A. H. Lumpkin, B. X. Yang, W. J. Berg, D. W. Rule, N. S. Sereno, and C. Y. Yao, AIP Conf. Proc. 868, 394 (2006).

[14] A. H. Lumpkin, W. J. Berg, N. S. Sereno, D. W. Rule, and C. Y. Yao, Phys. Rev. ST Accel. Beams 10, 022802 (2007).

[15] D. Rule and R. Fiorito, AIP Conf. Proc. 229, 315 (1991).

[16] V. A. Lebedev, Nucl. Instrum. Methods Phys. Res., Sect. A 372, 344 (1996).

[17] X. Artru, R. Chehab, K. Honkavaara, and A. Variola, Nucl. Instrum. Methods Phys. Res., Sect. B 145, 160 (1998).

[18] M. Castellano and V. A. Verzilov, Phys. Rev. ST Accel. Beams 1, 062801 (1998).

[19] Dao Xiang and Wen-Hui Huang, Nucl. Instrum. Methods Phys. Res., Sect. A 570, 357 (2007).

[20] M.L. Ter-Mikaelian, High-Energy Electromagnetic Processes in Condensed Media (Wiley/Interscience, New York, 1972).

[21] M. Born and E. Wolf, Principles of Optics, 7th Edition (Cambridge University Press, Cambridge, England, 1999).

[22] Dao Xiang and Wen-Hui Huang, Nucl. Instrum. Methods Phys. Res., Sect. B 248, 163 (2006).

[23] I. S. Gradsbteyn and I. M. Ryzbik, Table of Integrals, Series and Products (Academic Press, New York, 2000), 6th ed.

[24] D. W. Rule, R. B. Fiorito, and W. D. Kimura, AIP Conf. Proc. 390, 510 (1997).

[25] R. B. Fiorito, D. W. Rule, and W. D. Kimura, AIP Conf. Proc. 472, 725 (1999).

[26] B. H. Richardson, J. Opt. Soc. Am. 62, 55 (1972).

[27] L. B. Lucy, Astron. J. 79, 745 (1974).

[28] A. Potylitsyn, G. Naumenko, A. Aryshev, Y. Fukui, D. Cline, F. Zhou, M. Ross, P. Bolton, J. Urakawa, T. Muto, M. Tobiyama, R. Hamatsu, and P. Karataev, Nucl. Instrum. Methods Phys. Res., Sect. B 227, 170 (2005).

[29] Dao Xiang and Wen-Hui Huang, Phys. Rev. ST Accel. Beams 10, 012801 (2007). 